\title{
Моделирование переходных процессов в полупроводниковых приборах на основе 4H-SiC (учет неполной ионизации легирующих примесей в модуле ATLAS программного пакета SILVACO TCAD)
}

\author{
(C) П.А. Иванов, А.С. Потапов, Т.П. Самсонова
}

Физико-технический институт им. А.Ф. Иоффе Российской академии наук, 194021 Санкт-Петербург, Россия

E-mail: Pavel.Ivanov@mail.ioffe.ru

(Получена 25 октября 2018 г. Принята к печати 29 октября 2018 г.)

\begin{abstract}
Проведено моделирование переходного процесса в RC-цепи с обратно смещенным $4 H$-SiC $p-n$-диодом в качестве емкостного элемента. Для моделирования использовался программный модуль ATLAS, входящий в состав системы приборно-технологического проектирования SILVACO TCAD. Предложен и опробован альтернативный имеющемуся в ATLAS способ задания параметров легирующих примесей, частично ионизированных в $4 H$-SiC при комнатной температуре. (Имеющаяся в модуле ATLAS физическая модель INCOMPLETE, описывающая неполную ионизацию легирующих примесей в полупроводниках, непригодна для моделирования динамических характеристик приборов). Результаты моделирования обсуждаются в свете полученных ранее результатов экспериментальных исследований.
\end{abstract}

DOI: 10.21883/FTP.2019.03.47295.9014

\section{1. Введение}

В настоящее время для математического моделирования в полупроводниковой электронике широко используют программные системы приборно-технологического проектирования (TCAD), с помощью которых можно проводить сквозное моделирование приборов от процесса формирования их структуры до расчета их электрических характеристик. Одной из распространенных систем является программный пакет SILVACO TCAD [1]. В состав пакета SILVACO TCAD входит модуль ATLAS, предназначенный для полного анализа электрических характеристик готовых полупроводниковых приборов. Модуль ATLAS позволяет моделировать произвольно легированные полупроводниковые структуры, электрофизические свойства которых могут задаваться пользователем. Расчет переходных процессов в модуле ATLAS реализован с помощью симулятора электрических цепей (MixedMode), содержащих моделируемые полупроводниковые структуры и радиоэлектронные компоненты резисторы, конденсаторы, индуктивности, источники напряжения и тока, диоды, транзисторы и др. Одним из начальных этапов моделирования является задание параметров структуры прибора - толщин слоев, типов их проводимости и уровней легирования. Особенностью $4 H-\mathrm{SiC}$ является то, что акцепторные примеси (алюминий и бор) имеют большую энергию ионизации (0.2 и $0.4-0.6$ эВ соответственно [2]) и поэтому частично ионизированы при комнатной температуре. (Доноры - азот и фосфор - ионизированы почти на $100 \%$ ). Ранее было показано, что неполная ионизация акцепторов может существенным образом влиять на характеристики приборов на основе $\mathrm{SiC}$ с областями пространственного заряда (ОПЗ). В частности, в $\mathrm{SiC}$ $p$-типа проводимости имеет место „двойной“ эффеект экранирования внешнего электрического поля [3]: проникновение поля в $\mathrm{SiC}$ вызывает, помимо пространственного разделения частично ионизированных акцепторов и дырок, еще и дополнительную ионизацию акцепторов. При этом емкость приповерхностной области пространственного заряда (ОПЗ) в окрестности плоских зон возрастает как при обогащении, так и при обеднении приповерхностной области основными носителями - дырками (классическая теория поверхностной емкости полупроводника дает монотонное уменьшение поверхностной емкости при переходе от обогащения к обеднению). В работе [4] было показано, что в диодах Шоттки на основе $4 H-\mathrm{SiC}$ с имплантированными „борными“ охранными кольцами динамическое напряжение пробоя, измеряемое на импульсах микросекундной длительности, заметно увеличивается при уменьшении длительности импульсов. На качественном уровне это также было объяснено неполной ионизацией бора в $p$-областях планарных охранных $p-n$-переходов. На этих примерах видно, что при моделировании характеристик $4 H$-SiC-приборов учет неполной ионизации акцепторов весьма важен. Вообще говоря, в модуле ATLAS заложена модель INCOMPLETE, предназначенная для описания легирующих примесей с энергиями ионизации, задаваемыми пользователем. В работе [5] нами была предпринята попытка использовать эту модель для расчета переходного процесса в RC-цепи с обратносмещенным $4 H$-SiC $p^{+}-i-n^{+}$-диодом в качестве емкостного элемента. Полученные результаты были подвергнуты сомнениям в силу того, что модель INCOMPLETE никак не учитывает динамику ионизации легирующих примесей (в ней не предусмотрено задание таких важнейших параметров, как сечения захвата носителей примесями). В настоящей работе для моделирования переходных процессов в приборах на основе $4 H-\mathrm{SiC}$ предложен и 
опробован альтернативный способ задания параметров легирующих примесей. Обсуждается адекватность предложенного способа в свете полученных ранее экспериментальных результатов.

\section{2. Детали моделирования}

В качестве тестового объекта для моделирования был выбран $p^{++}-p-n_{o}-n^{++}$-диод, рассчитанный на стационарное напряжение пробоя $\sim 1000$ B. $p^{++}$и $n^{++}$-слои имеют одинаковую толщину и уровень легирования: 1 мкм и $10^{19} \mathrm{~cm}^{-3}$ соответственно. $p$ - и $n_{o}$-слои тоже имеют одинаковую толщину 10 мкм. Уровни легирования в $p$ - и $n_{o}$-слоях составляют $2 \cdot 10^{17}$ и $2 \cdot 10^{16} \mathrm{~cm}^{-3}$ соответственно. Для простоты примеси в $p^{++}, n_{o^{-}}$и $n^{++}$-областях считались полностью ионизированными, поэтому концентрации доноров и акцепторов в них задавались обычным способом - оператором DOPING с двумя параметрами: 1) тип примесей (n.type или p.type) и 2) концентрация (CONC). Акцепторы в p-слое описывались иначе - как глубокие ловушки. В модуле ATLAS ловушки описываются оператором TRAP с шестью параметрами: 1) тип ловушек (DONOR или ACCEPTOR), 2) энергетическое положение в запрещенной зоне (E.LEVEL), 3) концентрация (DENSITY), 4) $g$-фактор (DEGEN.FAC), 5) сечение захвата электрона (SIGN), 6) сечение захвата дырки (SIGP). В соответствии с принятыми в операторе TRAP правилами задания энергетического положения ловушек величина E.LEVEL для акцепторных примесей должна задаваться следующим образом (рис. 1): E.LEVEL $=E_{g}-\Delta E_{A}$, где $E_{g}-$ ширина запрещенной зоны, $\Delta E_{A}-$ энергия ионизации акцепторных примесей.

При моделировании предполагалось, что $p$-слой легирован бором. Бор создает в $4 H-\mathrm{SiC}$ два акцепторных уровня: мелкий с энергией ионизации 0.4 э и более глубокий ( $D$-центр) с энергией ионизации 0.6 эВ. Мелкий акцепторный уровень связывают с атомами бора, замещающими атомы кремния в решетке $4 H-\mathrm{SiC}$. $D$-центр представляет собой ассоциат бора (находящийся в кремниевой подрешетке) с вакансией углерода. $\mathrm{B}$ настоящей работе энергия ионизации акцепторных атомов бора была принята равной 0.6 эВ. (В работе [6] было показано, что при внедрении бора в слабо легированный $4 H$-SiC $n$-типа с помощью имплантации и (или) диффузии $p-n$-переход образуется за счет перекомпенсации доноров $D$-центрами). Характерное время $(\tau)$ термической ионизации $D$-центров при комнатной температуре ранее было оценено величиной $~ 100$ мкс [4]. В соответствии с известным выражением для времени термической ионизации глубоких акцепторных ловушек

$$
\tau=\frac{\exp \left(\frac{\Delta E_{A}}{k T}\right)}{\sigma v N_{v}}
$$

сечение захвата дырок $(\sigma)$ акцепторными атомами бора в настоящей работе было принято равным $10^{-13} \mathrm{~cm}^{2}$

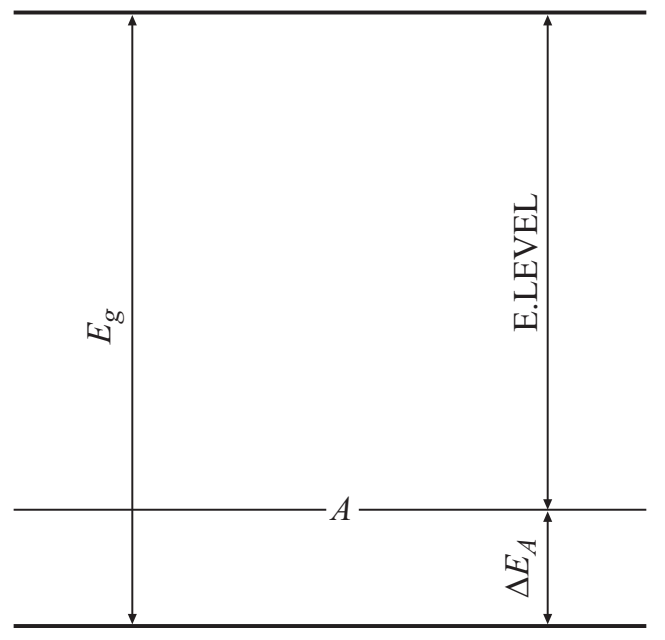

Рис. 1. Задание энергетического положения (E.LEVEL) акцепторных примесей.

$\left(v\right.$ - тепловая скорость дырок, $N_{v}-$ эффективная плотность состояний в валентной зоне $4 H-\mathrm{SiC})$.

Из физических моделей ATLAS с задаваемыми пользователем параметрами при моделировании использовалась аналитическая модель подвижности дырок и электронов (ANALYTIC). Параметры для этой модели были взяты из работы [7]. Площадь диодов была выбрана равной 0.25 мм² $^{2}$ Модельный эксперимент состоял в следующем. В момент времени $t=0$ на диод с последовательно включенным сопротивлением $R=50$ Ом подавалась ступень напряжения амплитудой 1000 В. Рассчитывалась временна́я зависимость напряжения на диоде. Кроме того, записывался стандартный файл data.str, coдержащий, в частности, профили распределения электрического поля в структуре диода в разные моменты времени в течение переходного процесса. Задачей моделирования было проследить динамику расширения ОПЗ после приложения обратного напряжения к диоду.

\section{3. Результаты и обсуждение}

На рис. 2 показаны рассчитанные временны́е зависимости напряжения на $p^{++}-p-n_{o}-n^{++}$-диоде. Кривая 1 получена для гипотетического случая полной ионизации акцепторов в р-области (в этом случае параметры акцепторов задавались оператором DOPING). Как видно, время переходного процесса, в течение которого напряжение на диоде поднимается до полной амплитуды $1000 \mathrm{~B}$, составляет около 1 нс. Кривая 2 рассчитана для случая неполной ионизации, когда параметры акцепторной примеси бора в р-области заданы оператором TRAP. Как видно, полное время нарастания напряжения на диоде оказывается приблизительно вдвое меньше по сравнению со случаем полной ионизации.

На рис. 3 показаны координатные распределения электрического поля в структуре $p^{++}-p-n_{o}-n^{++}$-диода 


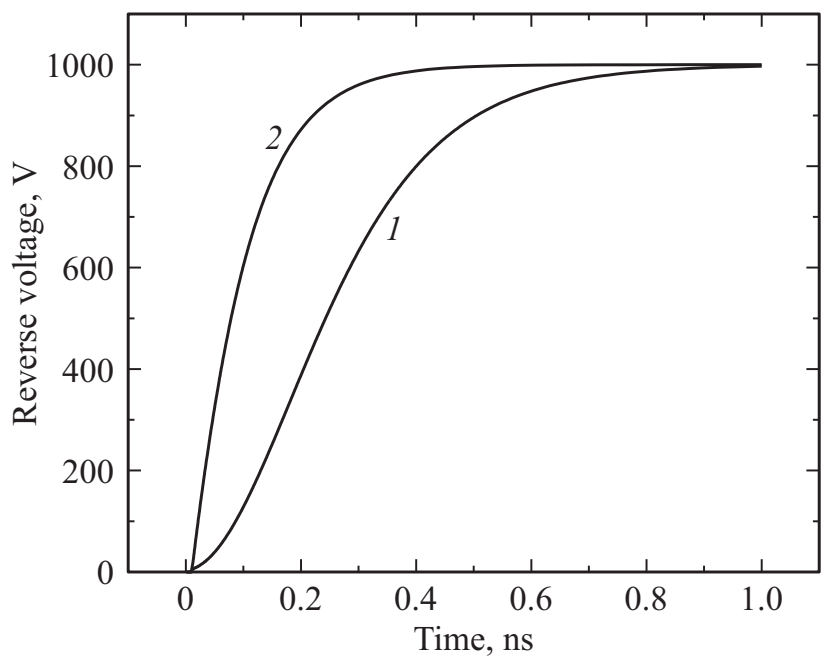

Рис. 2. Временные зависимости напряжения на $p^{++}-p-n_{o}-n^{++}$диоде: 1 - гипотетический случай полной ионизации; 2 - с учетом неполной ионизации.

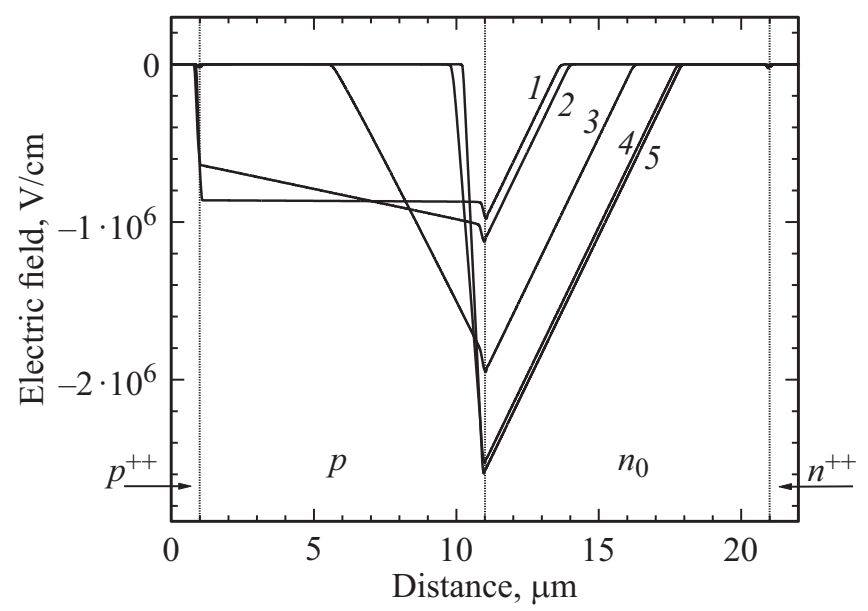

Рис. 3. Распределение электрического поля в структуре диода в разные моменты времени $t: 1-1 \mathrm{Hc}, 2-1$ мкс, $3-10$ мкс, $4-100$ мкс, $5-$ гипотетический случай полной ионизации $(t=1 \mathrm{Hc})$.

в разные моменты времени $t$. Кривая 5 соответствует времени $t=1 \mathrm{Hc}$ и получена для гипотетического случая полной ионизации. Как и ожидалось, вследствие асимметричного легирования $p-n_{o}$-перехода ОПЗ практически целиком сосредоточена в слабо легированной $n_{o}$-области. Кривая 1 тоже соответствует времени $t=1$ нс, но рассчитана для случая неполной ионизации, когда параметры акцепторной примеси бора в $p$-области заданы оператором TRAP. В данном случае ОПЗ в основном сосредоточена уже не в $n_{o^{-}}$, а в $p$-области из-за неполной ионизации бора. Отметим, что максимальное электрическое поле в структуре диода (в плоскости $p-n_{o}$-перехода) оказывается при этом в 2 раза меньше по сравнению со случаем полной ионизации. Так как ожидаемое время термической ионизации бора состав- ляет $\sim 100$ мкс, нестационарное распределение поля, показанное кривой 1, должно сохраняться в течение относительно длительного промежутка времени. Действительно, заметное перераспределение электрического поля начинается по истечении времени $\sim 1$ мкс (кривая 2). Далее по мере ионизации бора толщина ОПЗ в $p$-области сокращается (а в $n_{o}$-области, напротив, увеличивается) при одновременном увеличении величины максимального электрического поля (кривая 3). К моменту времени $t=100$ мкс распределение поля становится точно таким же, как и в случае полной ионизации (кривая 4). Продемонстрированная на рис. 3 динамика максимального электрического поля в плоскости $p-n_{o}$-перехода подтверждает выводы работы [4] о том, что за наблюдаемое увеличение напряжения пробоя имплантированных бором $p-n$-переходов при уменьшении длительности импульсов ответственна неполная ионизация бора в $p$-областях. Действительно, на коротких импульсах величина максимального электрического поля в структуре диода меньше стационарного значения, и именно поэтому напряжение пробоя на коротких импульсах оказывается больше своего стационарного значения.

\section{4. Заключение}

Заложенная в модуле ATLAS современного пакета SILVACO TCAD физическая модель INCOMPLETE не учитывает динамику ионизации легирующих примесей и поэтому не может давать адекватные результаты при моделировании динамических характеристик приборов на основе $4 \mathrm{H}$-SiC. В работе предложен и опробован альтернативный способ задания параметров легирующих примесей средствами ATLAS: параметры примесей предложено задавать оператором TRAP, предназначенным для описания глубоких ловушек. Проведенный модельный эксперимент показал, что данным способом становится возможным адекватное описание переходных процессов в 4H-SiC-приборах. Недостатком предложенного способа остается невозможность задания сложных профилей легирования, поэтому модернизация модуля ATLAS остается актуальной задачей для разработчиков данного программного продукта.

Исследование выполнено при финансовой поддержке гранта Российского научного фонда (проект № 14-2900094).

\section{Список литературы}

[1] http://www.silvaco.com

[2] G. Pensl, F. Ciobanu, T. Frank, M. Krieger, S. Reshanov, F. Schmid, M. Weidner. Int. J. High Speed Electron. Syst., 15, 705 (2005).

[3] Л.Б. Елфимов, П.А. Иванов. ФТП, 28, 161 (1994).

[4] П.А. Иванов, И.В. Грехов, А.С. Потапов, Т.П. Самсонова. ФТП, 42, 878 (2008). 
[5] П.А. Иванов, А.С. Потапов, И.В. Грехов. ЖТФ, 88, (6), 955 (2018).

[6] П.А. Иванов, А.С. Потапов, Т.П. Самсонова, О. Korol'kov, N. Sleptsuk. ФТП, 45, 1358 (2011).

[7] Т.Т. Мнацаканов, М.Е. Левинштейн, Л.И. Поморцева, С.Н. Юрков. ФТП, 38, 56 (2004).

Редактор А.Н. Смирнов

\section{Simulation of transient processes \\ in $4 \mathrm{H}$-SiC based semiconductor devices (the description of incomplete ionization of dopants in module ATLAS of SILVACO TCAD)}

P.A. Ivanov, A.S. Potapov, T.P. Samsonova

loffe Institute

194021 St. Petersburg, Russia

Abstract Transient processes in RC-circuit containing reversebiased $4 H-\mathrm{SiC}$ as a capacitor were simulated. The module ATLAS of SILVACO TCAD was used for simulations. An alternative way to set the parameters of partly ionized dopants in $4 H$-SiC is suggested. (The model INCOMPLETE available in ATLAS can not be used for simulations of transient processes in semiconductors due to that the INCOMPLETE model provides no carrier capture cross-sections). The simulation results obtained are discussed and compared with previous experimental results. 